\title{
The Role of Protease-Activated Receptor 2 in Hepatocellular Carcinoma after Hepatectomy
}

\author{
Ming-Chao Tsai ${ }^{1,2}$, Chih-Che Lin ${ }^{3}$, Ding-Wei Chen ${ }^{4}$, Yueh-Wei Liu ${ }^{3}$, Yi-Ju Wu ${ }^{3}$, Yi-Hao Yen ${ }^{1}$, \\ Pao-Yuan Huang ${ }^{1}\left(\mathbb{D}\right.$, Chih-Chien Yao ${ }^{1}$, Ching-Hui Chuang ${ }^{5}{ }^{(\mathbb{D}}$ and Chang-Chun Hsiao ${ }^{6, * \mathbb{C}}$
}

1 Division of Hepato-Gastroenterology, Department of Internal Medicine, Kaohsiung Chang Gung Memorial Hospital and Chang Gung University College of Medicine, Kaohsiung 83301, Taiwan; tony0779@gmail.com (M.-C.T.); cassellyen@yahoo.com.tw (Y.-H.Y.); paoyuan813@gmail.com (P.-Y.H.); chihchienyao@gmail.com (C.-C.Y.)

2 Graduate Institute of Clinical Medical Sciences, Chang Gung University College of Medicine, Kaohsiung 83301, Taiwan

3 Liver Transplantation Center and Department of Surgery, Kaohsiung Chang Gung Memorial Hospital and Chang Gung University College of Medicine, Kaohsiung 83301, Taiwan; immunologylin@gmail.com (C.-C.L.); anthony0612@me.com (Y.-W.L.); wuyiju0904@gmail.com (Y.-J.W.)

4 Center for Translational Research in Biomedical Sciences, Liver Transplantation Program and Department of Surgery, Kaohsiung Chang Gung Memorial Hospital and Chang Gung University College of Medicine, Kaohsiung 83301, Taiwan; dennis8870@gmail.com

5 Department of Nursing, Meiho University, Pingtung 91202, Taiwan; Helen.ch.chuang@gmail.com

6 Graduate Institute of Clinical Medical Sciences, College of Medicine, Chang Gung University, Division of Pulmonary and Critical Care Medicine, Kaohsiung Chang Gung Memorial Hospital, 123, Ta-Pei Road, Niao-Sung District, Kaohsiung 833, Taiwan

check for updates

Citation: Tsai, M.-C.; Lin, C.-C.; Chen, D.-W.; Liu, Y.-W.; Wu, Y.-J.; Yen, Y.-H.; Huang, P.-Y.; Yao, C.-C.; Chuang, C.-H.; Hsiao, C.-C. The Role of Protease-Activated Receptor 2 in Hepatocellular Carcinoma after Hepatectomy. Medicina 2021, 57, 574. https://doi.org/10.3390/ medicina57060574

Academic Editor: Giovanni Tarantino

Received: 27 April 2021

Accepted: 2 June 2021

Published: 4 June 2021

Publisher's Note: MDPI stays neutral with regard to jurisdictional claims in published maps and institutional affiliations.

Copyright: (c) 2021 by the authors. Licensee MDPI, Basel, Switzerland. This article is an open access article distributed under the terms and conditions of the Creative Commons Attribution (CC BY) license (https:// creativecommons.org/licenses/by/ $4.0 /)$.
* Correspondence: cchsiao@mail.cgu.edu.tw; Tel.: +886-7-7317123 (ext. 8979) or +886-955906053; Fax: +886-7-7311696

Abstract: Background and Objectives: Protease activated receptor-2 (PAR2) is elevated in a variety of cancers and has been promoted as a potential therapeutic target. However, the clinical and prognostic values of PAR2 in hepatocellular carcinoma (HCC) are poorly characterized. This study aimed to evaluate the expression of PAR2 in HCC tissues and examine the prognostic value of PAR2 after resection in HCC. Materials and Methods: Two hundred and eight resected specimens were collected from HCC patients at Kaohsiung Chang Gung Memorial Hospital. PAR2 protein expression was assessed by western blotting in HCC tissues and matched normal tissues. The correlation between PAR2 expression and clinicopathological parameters was analyzed. Disease-free survival (DFS) and overall survival (OS) were compared using the log-rank test. A Cox regression model was used to identify independent prognostic factors. Results: PAR2 was expressed at higher levels in HCC tissues than the paired adjacent nontumor tissues. High expression of PAR2 was associated with advanced tumor, node, metastasis (TNM )stage and histological grade. Kaplan-Meier analysis indicated high PAR2 expression was associated with poorer DFS and OS compared to low PAR2 expression. Multivariate analyses indicated high PAR2 expression [hazard ratio (HR), 1.779, $p=0.006$ ), $\alpha$-fetoprotein (AFP) $(\mathrm{HR}, 1.696, p=0.003)$, liver cirrhosis $(\mathrm{HR}, 1.735, p=0.002)$, and advanced TNM stage (HR, 2.061, $p<0.001)$ were prognostic factors for DFS, and advanced TNM stage (HR, 2.741, $p<0.001)$ and histological grade (HR, 2.675, $p=0.002)$ and high PAR2 expression (HR, 1.832, $p=0.012)$ were significant risk factors for OS. In subgroup analyses, the combination of PAR2 expression and serum AFP provided improved prognostic ability for OS and DFS. Conclusion: Combination PAR2 and AFP predict HCC outcomes after resection. PAR2 represents a potentially clinically relevant biomarker for HCC.

Keywords: PAR2; hepatocellular carcinoma; liver resection; AFP 


\section{Introduction}

Hepatocellular carcinoma (HCC), the sixth most common tumor type, is a major cause of cancer-related deaths around the world [1,2]. Although a number of therapeutic options exist, including liver transplantation, hepatectomy, and ablation, overall survival is still poor due to the high rate of recurrence (59-60\%) [3]. Several factors are prognostic for recurrence and/or survival after resection in HCC, including tumor size and differentiation, serum $\alpha$-fetoprotein (AFP), microvascular invasion, cirrhosis, surgical margin and metabolic syndrome [4-6]. However, more effective prognostic biomarkers need to be identified to improve the prediction of outcomes in HCC. The ability to identify patients at high risk of recurrence would enable clinicians to provide more intensive surveillance and detect recurrence at an earlier stage, when curative therapy may still be possible.

Protease-activated receptors (PARs) is a G-protein-coupled receptor that is activated by proteolytic cleavage of their extracellular $\mathrm{N}$ terminal domain [7]. It is also activated by trypsin, mast cell tryptase, and the tissue factor/factor VIIa and factor Xa complex [8]. A couple of studies demonstrated that PARs were associated with the modulation of vascular, inflammatory response, fibrogenesis, and carcinogenesis, which made PARs the potential targets for innovative therapies development [9-11]. In the setting of liver cancer, it could be demonstrated that PAR2 is expressed in HCC tissues, and in HCC cell lines, PAR2 can stimulate cell migration and invasion through different signaling pathways [12,13]. Hence, a crucial role of PAR2 in HCC progression can be hypothesized. However, the expression, function and clinical value of PAR2 in HCC have not been investigated. This study was designed to determine the expression of PAR2 in HCC tissues and examine the prognostic value of PAR2 after resection in HCC.

\section{Materials and Methods}

\subsection{Ethics}

This study was performed in accordance with the Declaration of Helsinki and approved by the Institutional Review Board of Chang Gung Memorial Hospital (IRB number: 201800049B0; Date: from August 2018 to July 2019). The ethics committee waived the requirement for informed consent; all patient data were anonymized.

\subsection{Tissue Specimens and Clinical Data}

Samples from 208 patients with HCC who underwent curative hepatic resection at Kaohsiung Chang Gung Memorial Hospital, Taiwan, between January 2008 and December 2015, were obtained from Tissue Bank, Kaohsiung Chang Gung Memorial Hospital. The samples included 208 paired HCC tumor/non-tumor tissues.

The resected tumors were histologically diagnosed according to international guidelines [14-18]. Disease-free survival (DFS) was calculated from the day of surgery until detection of recurrent or metastatic HCC by liver computed tomography (CT) or magnetic resonance imaging (MRI); overall survival (OS), from surgery to death, last contact, or December 2018. Survival outcomes were assessed using the patients' final medical records; data on patient demographic and clinical characteristics were also extracted from medical records, including age, gender, clinical stage (defined by the American Joint committee on Cancer tumor, node, metastasis (TNM) staging system [19]), tumor differentiation (according to the Edmondson-Steiner system [20]), number of tumors, maximal tumor size (according to the postoperative pathology), pathological microvascular invasion, serum hepatitis markers (HBsAg and anti-HCV), serum alpha-fetoprotein (AFP) level, and cirrhosis (Ishak score of 5 or 6 [21]).

\subsection{Western Blotting}

Tissue samples $(50 \mathrm{mg}$ ) were homogenized in lysis buffer $(40 \mathrm{mmol} / \mathrm{L} \mathrm{N}$-2-hydroxyethylpiperazine $N^{\prime}$-2-ethane sulphonate, $1 \%$ Triton X-100, 10\% glycerol, $1 \mathrm{mmol} / \mathrm{L}$ phenylmethanesulfonyl fluoride), sonicated, centrifuged at $12,000 \mathrm{rpm}$ for $30 \mathrm{~min}$ at $4{ }^{\circ} \mathrm{C}$ and the total protein extracts $(25-50 \mu \mathrm{g} / \mathrm{lane})$ were subjected to immunoblot analysis. Proteins were transferred 
onto polyvinylidene di-fluoride membranes (Immobilon-P membrane; Millipore, Bedford, MA, USA), incubated with anti-PAR2 antibody (ab180953, Abcam, Cambridge, UK) overnight at $4{ }^{\circ} \mathrm{C}$, washed, incubated with anti-rabbit and anti-mouse IgG secondary peroxidase-conjugated antibodies at $25^{\circ} \mathrm{C}$ for $1 \mathrm{~h}$ (Cell Signaling, Danvers, MA, USA), and the bands were developed using the ECL plus chemiluminescence kit (Amersham Pharmacia Biotech, Buckinghamshire, UK). Target protein expression values were normalized to $\beta$-actin. Two-fold higher PAR2 protein expression in the tumor sample compared to the matched nontumor tissue was defined as high PAR2 expression.

\subsection{Statistical Analyses}

Data were analyzed using SPSS version 22 (IBM, Chicago, IL, USA) and expressed as median (interquartile range, IQR) or mean \pm standard deviation. Categorical data were compared using the chi-square test. DFS and OS were assessed using the Kaplan-Meier method and compared using the log-rank test. The Cox proportional hazards model was used to identify independent risk factors for DFS and OS; factors with a $p$-value $<0.3$ in univariate analysis were included in the multivariate analysis. Two-sided $p$-values $<0.05$ were considered significant.

\section{Results}

\subsection{Patient Characteristics}

The clinicopathological features of the 208 patients with HCC who underwent curative resection are summarized in Table 1 . Overall, the cohort included 162 males $(78 \%)$ and 46 females (22\%), with a mean age of 59 years (range: $25-82)$. The etiology of HCC was associated with hepatitis B virus (HBV) in 120 cases (59.1\%) and hepatitis $C$ virus (HCV) in 63 cases $(31 \%)$; the remaining 25 cases $(12 \%)$ had other/unknown etiologies. The median largest tumor diameter was $5 \mathrm{~cm}$ (IQR $3.5-8 \mathrm{~cm}$ ). Pathological examination confirmed microvascular invasion in 105 cases (53.8\%). Mean follow-up duration was 58 months (range, 2-140 months). Of the 142 (68.3\%) patients who developed recurrence, the mean time to recurrence was 40.1 months. Overall, 112 patients (53.8\%) died during follow-up.

\subsection{Association between PAR2 and the Clinicopathological Features of HCC}

The expressions of PAR2 were examined by western blot analysis in 208 paired HCC and nontumor tissues (Figure 1). Compared with the paired nontumor tissues, high levels (defined as greater than twofold increase) of PAR2 expression in 143 of 208 $(68.8 \%)$ HCC cases, and the other $65(31.2 \%)$ were defined as PAR2 low expression group. The clinicopathological features of patients with HCC stratified by PAR2 expression are summarized in Table 2. We found that patients with high expression of PAR2 were associated with advanced tumor-node-metastasis (TNM) stage $(p=0.005)$, and relative high serum AFP level $(p=0.060)$, but not with other characteristics such as gender, age, etiology, tumor, size, vascular invasion, and pathological stage.

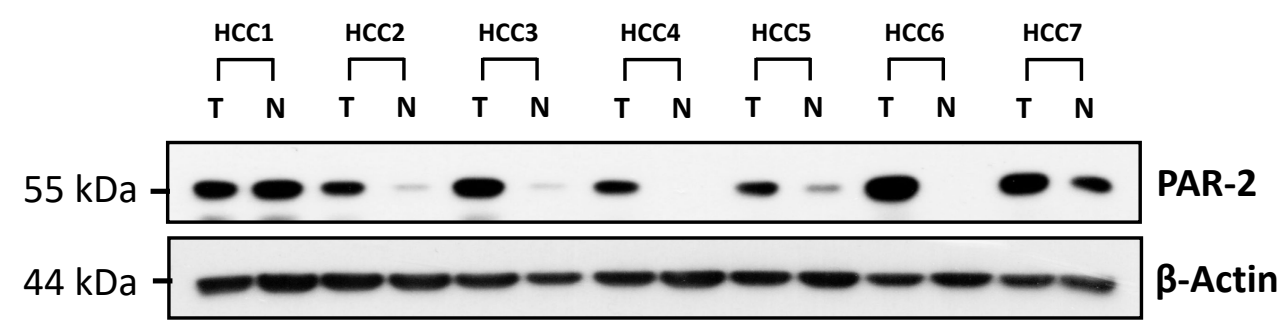

Figure 1. Western blot analysis of PAR2 in seven representative HCC tissue (T) and their paired non-tumor (N) tissue. $\beta$-Actin was used as a loading control. 
Table 1. Clinicopathological features of the 208 patients with HCC undergoing tumor resection.

\begin{tabular}{|c|c|}
\hline \multicolumn{2}{|c|}{ Clinicopathological Feature } \\
\hline Age [years, mean (SD)] & $59(13.5)$ \\
\hline Male gender, $n(\%)$ & $162(78)$ \\
\hline $\mathrm{AFP}[\mathrm{ng} / \mathrm{mL}$ median (IRQ)] & $48(5-825)$ \\
\hline $\mathrm{AFP}>15 \mathrm{ng} / \mathrm{mL}, n(\%)$ & $124(59.6)$ \\
\hline $\mathrm{AFP}>200 \mathrm{ng} / \mathrm{mL}, n(\%)$ & $77(37)$ \\
\hline Liver cirrhosis, $n(\%)$ & $108(51.9)$ \\
\hline Etiology & \\
\hline Hepatitis B & $120(59.1)$ \\
\hline Hepatitis C & $63(31)$ \\
\hline Other/unknown & $25(12)$ \\
\hline \multicolumn{2}{|c|}{ Tumor Characteristics } \\
\hline Tumor size $[\mathrm{cm}, \text { median }(\mathrm{IQR})]^{\mathrm{a}}$ & $5(3.5-8)$ \\
\hline Tumor size $>3 \mathrm{~cm}, n(\%)$ & $169(81.3)$ \\
\hline Tumor size $>5 \mathrm{~cm}, n(\%)$ & $103(49.5)$ \\
\hline Solitary tumor, $n(\%)$ & $166(79.8)$ \\
\hline Microvascular invasion, $n(\%)$ & $105(53.8)$ \\
\hline TNM stage (I:II:III:IV) & $44: 78: 54: 31$ \\
\hline \multicolumn{2}{|l|}{ Histological grade (well:moderate:poor) } \\
\hline Well & $41(19.7)$ \\
\hline Moderate & $130(62.5)$ \\
\hline Poor & $37(17.8)$ \\
\hline \multicolumn{2}{|c|}{ Clinical Outcome } \\
\hline HCC recurrence, $n(\%)$ & $142(68.3)$ \\
\hline Mean time to recurrence, [months, mean (SD)] & $40.1(43.1)$ \\
\hline Died, $n(\%)$ & $112(53.8)$ \\
\hline Mean time to death, [months, mean (SD)] & $58(44.6)$ \\
\hline
\end{tabular}

Table 2. Association between PAR2 and the clinicopathological features of HCC.

\begin{tabular}{|c|c|c|c|}
\hline \multirow{2}{*}{ Variable } & \multicolumn{2}{|c|}{ PAR2 Expression $(n=208)$} & \multirow{2}{*}{$p$ Value } \\
\hline & Low $(n=65)$ & High $(n=143)$ & \\
\hline Gender & & & 0.096 \\
\hline Female & $19(29.2)$ & $27(18.9)$ & \\
\hline Male & $46(70.8)$ & $116(81.1)$ & \\
\hline Age (years) & & & 0.155 \\
\hline$<60$ & $29(44.6)$ & $79(55.2)$ & \\
\hline$\geq 60$ & $36(55.4)$ & $64(44.8)$ & \\
\hline $\operatorname{AFP}(n g / m L)$ & & & 0.060 \\
\hline$<200$ & $47(72.3)$ & $84(58.7)$ & \\
\hline$\geq 200$ & $18(27.7)$ & $59(41.3)$ & \\
\hline HBsAg & & & 0.149 \\
\hline Negative & $30(48.4)$ & $53(37.6)$ & \\
\hline Positive & $32(51.6)$ & $88(62.4)$ & \\
\hline $\mathrm{HCV} \mathrm{Ab}$ & & & 0.803 \\
\hline Negative & $42(67.7)$ & $98(69.5)$ & \\
\hline Positive & $20(32.3)$ & $43(30.5)$ & \\
\hline Liver cirrhosis & & & 0.940 \\
\hline No & $31(47.7)$ & $69(48.3)$ & \\
\hline Yes & $34(52.3)$ & $74(51.7)$ & \\
\hline Tumor size (cm) & & & 0.121 \\
\hline$<5$ & $38(58.5)$ & $67(46.9)$ & \\
\hline$\geq 5$ & $27(41.5)$ & $76(53.1)$ & \\
\hline
\end{tabular}


Table 2. Cont.

\begin{tabular}{|c|c|c|c|}
\hline \multirow{2}{*}{ Variable } & \multicolumn{2}{|c|}{ PAR2 Expression $(n=208)$} & \multirow{2}{*}{$p$ Value } \\
\hline & Low $(n=65)$ & High $(n=143)$ & \\
\hline Tumor number & & & 0.963 \\
\hline Single & $52(80)$ & 114 (79.7) & \\
\hline Multiple & $13(20)$ & $29(20.3)$ & \\
\hline $\begin{array}{l}\text { Microvascular } \\
\text { invasion }\end{array}$ & & & 0.593 \\
\hline Absent & $28(49.1)$ & $62(44.9)$ & \\
\hline Present & $29(50.9)$ & $76(55.1)$ & \\
\hline TNM stage & & & 0.005 \\
\hline I or II & $47(73.4)$ & $75(52.4)$ & \\
\hline III or IV & $17(26.6)$ & $68(47.6)$ & \\
\hline Histological grade & & & 0.590 \\
\hline Well & $14(21.5)$ & $27(18.9)$ & \\
\hline Moderate & $42(64.6)$ & $88(61.5)$ & \\
\hline Poor & $9(13.9)$ & $28(19.6)$ & \\
\hline
\end{tabular}

Data are expressed as number (percentage). PAR2: Protease Activated Receptor-2; HBV: hepatitis B surface; HCV: hepatitis $C$ virus; AFP: alpha-fetoprotein; TNM: tumor node metastasis.

\subsection{Association between PAR2 and Recurrence in HCC}

After the median follow-up of 24 months (IQR, 6.3-60.3), 142/208 (68.3\%) patients had developed recurrence. The cumulative incidence of recurrence for patients with high PAR2 expression was $39.7 \%$ at year $1,65.3 \%$ at year 3 , and $72.7 \%$ at year 5 . In contrast, the cumulative incidence of recurrence was $22.0 \%$ at year $1,35.1 \%$ at year 3 , and $55.5 \%$ at year 5 for patients with low PAR2 expression $(p<0.001$; Table 3 and Figure 2A). In addition to PAR2 expression, serum AFP ( $\geq 200 \mathrm{ng} / \mathrm{mL} ; p<0.001)$, cirrhosis $(p=0.001)$, tumor size ( $\geq 5 \mathrm{~cm} ; p=0.001)$, number of tumors (multiple tumors; $p=0.006)$, vascular invasion $(p<0.001)$, advanced TNM stage $(p<0.001)$, and advanced histological grade $(p<$ 0.001 ) were associated with recurrence in HCC (Table 3$)$.

Table 3. Cumulative incidence of recurrence after resection for patients with HCC.

\begin{tabular}{|c|c|c|c|c|c|}
\hline Variable & Number of Patients & 1 Year $(\%)$ & 3 Years (\%) & 5 Years $(\%)$ & $p$-Value \\
\hline Age (years) & & & & & 0.062 \\
\hline$<60$ & 108 & 43.4 & 60.7 & 70.3 & \\
\hline$\geq 60$ & 100 & 24.3 & 48.1 & 64.3 & \\
\hline Gender & & & & & 0.201 \\
\hline Female & 46 & 26.7 & 49.8 & 64.3 & \\
\hline Male & 162 & 36.8 & 56.2 & 66.3 & \\
\hline $\begin{array}{l}\text { AFP } \\
(\mathrm{ng} / \mathrm{mL})\end{array}$ & & & & & $<0.001$ \\
\hline$<200$ & 131 & 22 & 45.4 & 60.9 & \\
\hline$\geq 200$ & 77 & 49.9 & 69.5 & 74.4 & \\
\hline HBsAg & & & & & 0.155 \\
\hline Negative & 88 & 26.7 & 48.7 & 61.4 & \\
\hline Positive & 120 & 36.5 & 58.6 & 67.8 & \\
\hline $\mathrm{HCV} \mathrm{Ab}$ & & & & & 0.530 \\
\hline Negative & 125 & 36 & 55.4 & 65.4 & \\
\hline Positive & 63 & 24.7 & 54.1 & 65.5 & \\
\hline $\begin{array}{l}\text { Liver } \\
\text { cirrhosis }\end{array}$ & & & & & 0.001 \\
\hline Absent & 100 & 27.2 & 48.8 & 56.1 & \\
\hline Present & 108 & 37 & 59.2 & 74.1 & \\
\hline $\begin{array}{l}\text { Tumor size } \\
(\mathrm{cm})\end{array}$ & & & & & 0.001 \\
\hline$<5$ & 105 & 21.1 & 45.5 & 58.3 & \\
\hline$\geq 5$ & 103 & 43.6 & 63.2 & 73.5 & \\
\hline
\end{tabular}


Table 3. Cont.

\begin{tabular}{|c|c|c|c|c|c|}
\hline Variable & Number of Patients & 1 Year (\%) & 3 Years $(\%)$ & 5 Years $(\%)$ & $p$-Value \\
\hline $\begin{array}{l}\text { Tumor } \\
\text { number }\end{array}$ & & & & & 0.006 \\
\hline Single & 166 & 30.1 & 51.2 & 61.2 & \\
\hline Multiple & 42 & 40.4 & 65.3 & 81.9 & \\
\hline $\begin{array}{l}\text { Microvascular } \\
\text { invasion }\end{array}$ & & & & & $<0.001$ \\
\hline Absent & 103 & 13 & 38 & 51.9 & \\
\hline Present & 105 & 52 & 73.1 & 82.4 & \\
\hline TNM stage & & & & & $<0.001$ \\
\hline $\mathrm{I} / \mathrm{II}$ & 123 & 17.9 & 40.7 & 53 & \\
\hline III/IV & 85 & 53 & 73.9 & 84.4 & \\
\hline $\begin{array}{l}\text { Histological } \\
\text { grade }\end{array}$ & & & & & 0.001 \\
\hline Well & 41 & 13.7 & 29.4 & 46.4 & \\
\hline Moderate & 130 & 44 & 60.1 & 69.4 & \\
\hline Poor & 37 & 47.9 & 65 & 76.7 & \\
\hline $\begin{array}{l}\text { PAR2 } \\
\text { expression }\end{array}$ & & & & & $<0.001$ \\
\hline Low & 65 & 22.0 & 35.1 & 55.5 & \\
\hline High & 143 & 39.7 & 65.3 & 72.7 & \\
\hline
\end{tabular}

Univariate analyses using the Cox proportional hazard model demonstrated that AFP $\geq 200 \mathrm{ng} / \mathrm{mL}$ (hazard ratio (HR), 1.675; 95\% CI, 1.284-2.385; $p<0.001$ ), liver cirrhosis (HR, 1.647; 95\% CI, 1.204-2.253; $p=0.002)$, tumor size $\geq 5 \mathrm{~cm}$ (HR, 1.668; 95\% CI, 1.228-2.266; $p=0.001)$, multiple tumors (HR, 1.603; 95\% CI, 1.14-2.254; $p=0.007)$, vascular invasion (HR, 2.625; 95\% CI, 1.902-3.622; $p<0.001$ ), TNM stage (III/IV vs. I/II; HR, 2.655; 95\% CI, 1.95-3.613; $p<0.001$ ), pathological grade (poor/moderate vs. well; HR, 1.95; 95\% CI, 1.304-2.916; $p=0.001$ ), and PAR2 expression (high vs. low; HR, 1.969; 95\% CI, 1.144-3.388; $p=0.014$ ) were associated with a significantly higher risk of recurrence. These significant covariates from univariate analysis were entered into multivariate Cox analysis. Serum $\mathrm{AFP} \geq 200 \mathrm{ng} / \mathrm{mL}(\mathrm{HR}, 1.696 ; 95 \% \mathrm{CI}, 1.196-2.403 ; p=0.003)$, liver cirrhosis (HR, 1.735; $95 \%$ CI, 1.221-2.466; $p=0.002$ ), TNM stage (III/IV vs. I/II; HR, 2.061; 95\% CI, 1.447-2.934; $p<0.001$ ), and PAR2 expression (high vs. low; HR, 1.779; 95\% CI, 1.181-2.681; $p=0.006$ ) were identified as independent risk factors for recurrence in HCC (Table 4).

Table 4. Univariate and multivariate analysis of factors associated with cumulative recurrence in HCC.

\begin{tabular}{|c|c|c|c|c|c|}
\hline \multirow{2}{*}{ Variable } & \multirow{2}{*}{ Comparison } & \multicolumn{2}{|c|}{ Univariate } & \multicolumn{2}{|c|}{ Multivariate } \\
\hline & & HR (95\% CI) & $p$-Value & HR (95\% CI) & $p$-Value \\
\hline Age (years) & $\geq 60$ vs. $<60$ & $0.749(0.551-1.018)$ & 0.065 & & \\
\hline Sex & Male vs. Female & $1.272(0.876-1.847)$ & 0.206 & & \\
\hline $\operatorname{AFP}(n g / m L)$ & $\geq 200$ vs. $<200$ & $1.675(1.284-2.385)$ & $<0.001$ & $1.696(1.196-2.403)$ & 0.003 \\
\hline HBV & Positive vs. Negative & $1.262(0.913-1.746)$ & 0.159 & & \\
\hline $\mathrm{HCV}$ & Positive vs. Negative & $0.896(0.635-1.265)$ & 0.534 & & \\
\hline Liver cirrhosis & Presence vs. Absence & $1.647(1.204-2.253)$ & 0.002 & $1.735(1.221-2.466)$ & 0.002 \\
\hline Tumor size $(\mathrm{cm})$ & $\geq 5$ vs. $<5$ & $1.668(1.228-2.266)$ & 0.001 & & \\
\hline Tumor number & Multiple vs. Single & $1.603(1.140-2.254)$ & 0.007 & & \\
\hline Microvascular invasion & Presence vs. Absence & $2.625(1.902-3.622)$ & $<0.001$ & & \\
\hline TNM stage & III + IV vs. I + II & $2.655(1.95-3.613)$ & $<0.001$ & $2.061(1.447-2.934)$ & $<0.001$ \\
\hline Histological grade & Poor/Moderate vs. Well & $1.95(1.304-2.916)$ & 0.001 & & \\
\hline PAR2 expression & High vs. Low & $1.969(1.144-3.388)$ & 0.014 & $1.779(1.181-2.681)$ & 0.006 \\
\hline
\end{tabular}




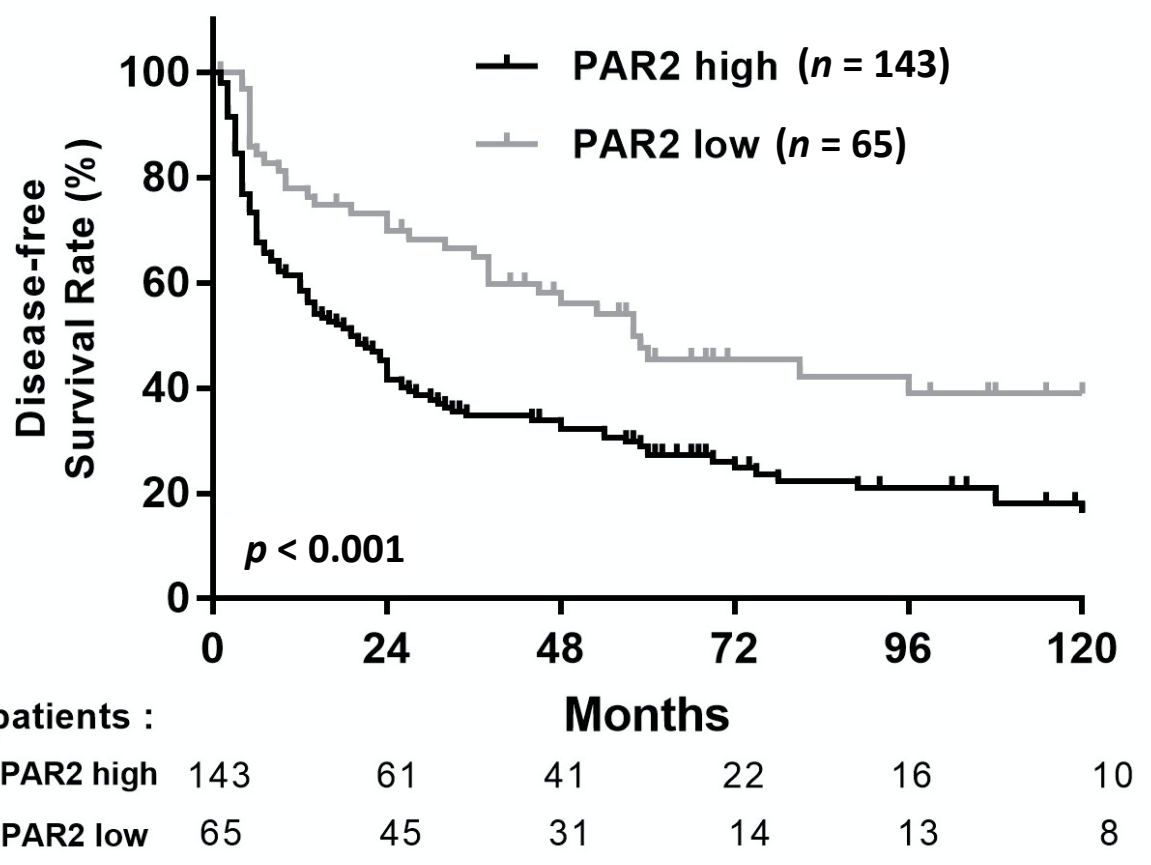

(A)

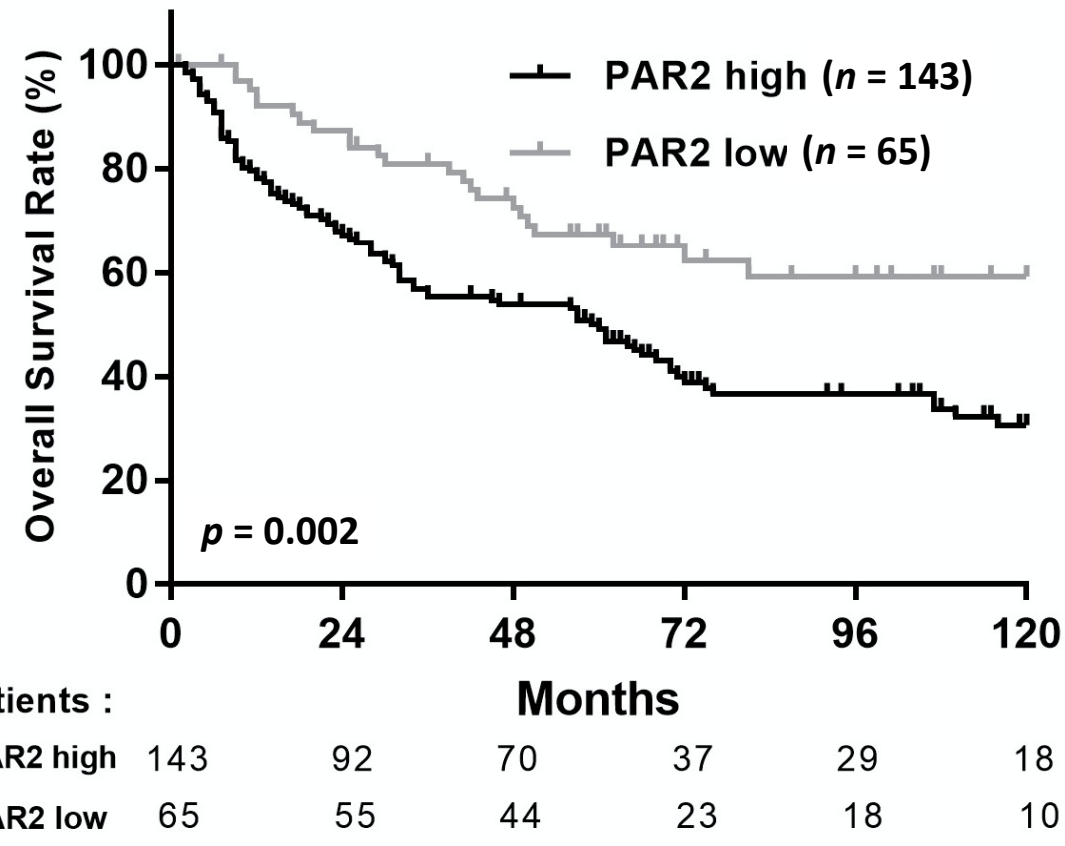

(B)

Figure 2. Disease-free survival (A) and overall survival (B) of HCC patients after resection stratified by PAR2 expression.

\subsection{Association between PAR2 and Overall Survival}

After the median follow-up of 57 months (IQR, 19-84), 112/208 (53.8\%) patients had died. The OS rates of patients with high PAR2 expression were $78.8 \%$ at year $1,56.9 \%$ at year 3 , and $49.9 \%$ at year 5 compared with $95.2 \%$ at year $1,80.9 \%$ at year 3 , and $67.3 \%$ at year 5 for patients with low PAR2 expression ( $p=0.002$; Table 5 and Figure 2B). In addition to Chibby, serum AFP $(\geq 200 \mathrm{ng} / \mathrm{mL} ; p=0.002)$, cirrhosis $(p<0.001)$, vascular invasion $(p<0.001)$, advanced TNM stage $(p<0.001)$, and advanced histological grade $(p<0.001)$ were associated with poorer OS after resection in patients with HCC. 
Table 5. Cumulative incidence of survival after resection in patients with HCC.

\begin{tabular}{|c|c|c|c|c|c|}
\hline Variable & Number of Patients & 1 Year $(\%)$ & 3 Years $(\%)$ & 5 Years $(\%)$ & $p$-Value \\
\hline Age (years) & & & & & 0.313 \\
\hline$<60$ & 108 & 81.7 & 59.8 & 50.9 & \\
\hline$\geq 60$ & 100 & 86.6 & 68.4 & 57.6 & \\
\hline Gender & & & & & 0.382 \\
\hline Female & 46 & 88.7 & 69.8 & 59.7 & \\
\hline Male & 162 & 81.1 & 62 & 52.9 & \\
\hline $\begin{array}{l}\text { AFP } \\
\text { (ng/mL) }\end{array}$ & & & & & 0.002 \\
\hline$<200$ & 131 & 88.2 & 72 & 61.4 & \\
\hline$\geq 200$ & 77 & 73.4 & 47.5 & 41.3 & \\
\hline $\mathrm{HBsAg}$ & & & & & 0.109 \\
\hline Negative & 88 & 84.1 & 70.3 & 61.7 & \\
\hline Positive & 120 & 81.3 & 57.6 & 48.9 & \\
\hline $\mathrm{HCV} \mathrm{Ab}$ & & & & & 0.472 \\
\hline Negative & 125 & 81.9 & 60.1 & 50.4 & \\
\hline Positive & 63 & 83.3 & 68.1 & 61.7 & \\
\hline $\begin{array}{l}\text { Liver } \\
\text { cirrhosis }\end{array}$ & & & & & 0.14 \\
\hline Absent & 100 & 84 & 65.1 & 57.6 & \\
\hline Present & 108 & 81.6 & 60.9 & 50.8 & \\
\hline $\begin{array}{l}\text { Tumor size } \\
(\mathrm{cm})\end{array}$ & & & & & $<0.001$ \\
\hline$<5$ & 105 & 94.1 & 74.9 & 62.9 & \\
\hline$\geq 5$ & 103 & 71.6 & 51.3 & 45.2 & \\
\hline Tumor no. & & & & & 0.227 \\
\hline Single & 166 & 84.1 & 66.1 & 56.8 & \\
\hline Multiple & 42 & 77.8 & 51.9 & 44.4 & \\
\hline $\begin{array}{l}\text { Vascular } \\
\text { invasion }\end{array}$ & & & & & $<0.001$ \\
\hline Absent & 103 & 95.4 & 82.7 & 72.9 & \\
\hline Present & 105 & 69 & 42.1 & 33.2 & \\
\hline TNM stage & & & & & $<0.001$ \\
\hline $\mathrm{I} / \mathrm{II}$ & 123 & 95.7 & 81.1 & 71 & \\
\hline III/IV & 85 & 64.5 & 37.7 & 30.6 & \\
\hline $\begin{array}{l}\text { Histological } \\
\text { grade }\end{array}$ & & & & & $<0.001$ \\
\hline Well & 41 & 96.1 & 86.1 & 79.9 & \\
\hline Moderate & 130 & 81.2 & 61.2 & 51.3 & \\
\hline Poor & 37 & 73.2 & 43.2 & 34.6 & \\
\hline $\begin{array}{l}\text { PAR2 } \\
\text { expression }\end{array}$ & & & & & 0.002 \\
\hline Low & 65 & 95.2 & 80.9 & 67.3 & \\
\hline High & 143 & 78.8 & 56.9 & 49.9 & \\
\hline
\end{tabular}

HBV: hepatitis B surface; HCV: hepatitis C virus; AFP: alpha-fetoprotein; IHC, immunohistochemistry; WB, western blotting.

Univariate analyses indicated serum AFP $\geq 200 \mathrm{ng} / \mathrm{mL}$ (HR, 1.721; 95\% CI, 1.221-2.426; $p=0.002)$, tumor size $\geq 5 \mathrm{~cm}(\mathrm{HR}, 2.083 ; 95 \% \mathrm{CI}, 1.469-2.955 ; p<0.001)$, presence of microvascular invasion ( $\mathrm{HR}, 3.231 ; 95 \% \mathrm{CI}, 2.122-4.696 ; p<0.001)$, advanced TNM stage (III/IV vs. I/II; HR, 3.356; 95\% CI, 2.366-4.761; $p<0.001$ ), pathological grade (poor/moderate vs. well; HR, 2.946; 95\% CI, 1.719-5.605; $p<0.001$ ), and PAR2 expression (high vs. low; HR, 2.027; 95\% CI, 1.29-3.184; $p=0.002$ ) were associated with significantly poorer OS. Multivariate Cox analysis of the significant covariates from univariate analyses revealed that TNM stage (III/IV vs. I/II; HR, 2.747; 95\% CI, 1.851-4.077; $p<0.001$ ), pathological stage (poor/moderate vs. well; HR, 2.675; 95\% CI, 1.417-5.051; $p=0.002$ ), and PAR2 expression (high vs. low; HR, 1.832; 95\% CI, 1.142-2.938; $p=0.012$ ) were significant independent prognostic factors for OS (Table 6). 
Table 6. Univariate and multivariate analysis of factors associated with overall mortality in HCC.

\begin{tabular}{|c|c|c|c|c|c|}
\hline \multirow{2}{*}{ Variable } & \multirow{2}{*}{ Comparison } & \multicolumn{2}{|c|}{ Univariate } & \multicolumn{2}{|c|}{ Multivariate } \\
\hline & & HR $(95 \%$ CI) & $p$-Value & HR (95\% CI) & $p$-Value \\
\hline Age (Years) & $\geq 60$ vs. $<60$ & $0.938(0.595-1.182)$ & 0.315 & & \\
\hline Sex & Male vs. Female & $1.203(0.793-1.826)$ & 0.384 & & \\
\hline $\operatorname{AFP}(n g / m L)$ & $\geq 200$ vs. $<200$ & $1.721(1.221-2.426)$ & 0.002 & & \\
\hline HBV & Positive vs. Negative & $1.346(0.934-1.941)$ & 0.111 & & \\
\hline $\mathrm{HCV}$ & Positive vs. Negative & $0.869(0.591-1.276)$ & 0.474 & & \\
\hline Liver cirrhosis & Present vs. Absent & $1.295(0.917-1.828)$ & 0.143 & & \\
\hline Tumor size $(\mathrm{cm})$ & $\geq 5$ vs. $<5$ & $2.083(1.469-2.955)$ & $<0.001$ & & \\
\hline Tumor number & Multiple vs. Single & $1.293(0.859-1.888)$ & 0.229 & & \\
\hline Microvascular invasion & Present vs. Absent & $3.231(2.122-4.696)$ & $<0.001$ & & \\
\hline TNM stage & III + IV vs. I + II & $3.356(2.366-4.761)$ & $<0.001$ & $2.747(1.851-4.077)$ & $<0.001$ \\
\hline Histological grade & Poor/Moderate vs. Well & $2.946(1.719-5.05)$ & $<0.001$ & $2.675(1.417-5.051)$ & 0.002 \\
\hline PAR2 expression & High vs. Low & $2.027(1.29-3.184)$ & 0.002 & $1.832(1.142-2.938)$ & 0.012 \\
\hline
\end{tabular}

\subsection{Prognostic Value of Chibby Combined with Serum AFP}

As serum AFP $\geq 200 \mathrm{ng} / \mathrm{mL}$ was prognostic for poorer DFS and OS, we explored whether the prognostic value of PAR2 varied with serum AFP. The patients were divided into four subgroups: high PAR2 and AFP $\geq 200 \mathrm{ng} / \mathrm{mL}(n=59)$; high PAR2 and AFP $<200 \mathrm{ng} / \mathrm{mL}(n=84) ;$ low PAR2 and AFP $\geq 200 \mathrm{ng} / \mathrm{mL}(n=18) ;$ and low PAR2 and AFP $<200 \mathrm{ng} / \mathrm{mL}(n=47)$. Patients with low PAR2 and AFP $<200 \mathrm{ng} / \mathrm{mL}$ had significantly better DFS ( $p<0.001$, Figure 3A) and OS ( $p<0.001$, Figure 3B) rates than all other subgroups. Among patients with serum AFP $<200 \mathrm{ng} / \mathrm{mL}$, the low PAR2 subgroup achieved significantly better DFS ( $p=0.024$, Figure 3 A) and OS ( $p=0.058$, Figure 3B) than the high PAR2 subgroup. Similarly, among patients with AFP $\geq 200 \mathrm{ng} / \mathrm{mL}$, the low PAR2 subgroup had significantly better DFS ( $p=0.007$, Figure $3 \mathrm{~A})$ and OS $(p=0.014$, Figure $3 \mathrm{~B})$ than the high PAR2 subgroup.

\section{Discussion}

Developments in surgical techniques and new targeted drugs have markedly improved the treatment of HCC in recent years [22]. However, outcomes remain poor due to the high rate of recurrence after resection. Although several biomarkers have prognostic value for outcome after surgery, more effective novel biomarkers and targets for HCC urgently need to be identified.

PAR2, a member of the G-protein coupled receptor 1 family [7], is expressed in a wide range of cellular types, where it has been involved in multiple physiological and pathophysiological processes including cancers [23,24]. Although PAR2 has been implicated in the pathogenesis of several cancers, including lung, skin, stomach, ovary, kidney and colon [25-29], and mounting in vitro studies suggested a role for PAR2 in cancer development, including cell proliferation, invasion, and metastasis [30,31], only a limited number of studies of PAR2 in the HCC are currently available. In this study, we used western blot analysis to confirm that PAR2 is upregulated in the majority of HCC tissues (up to 70\%). Moreover, upregulation of PAR2 was significantly associated with advanced TNM stage and poor differentiation, and PAR2 tumor expression was an independent prognostic factor for OS and DFS after resection. To our best of our knowledge, this is the largest cohort to evaluate the expression of PAR2 in predicting the outcomes of HCC after resection. Therefore, we confirm that assessment of PAR2 expression could potentially help to predict the outcome of patients with HCC; patients with high expression of PAR2 may benefit from more intensive surveillance and timely adjuvant treatment to improve their prognosis. 


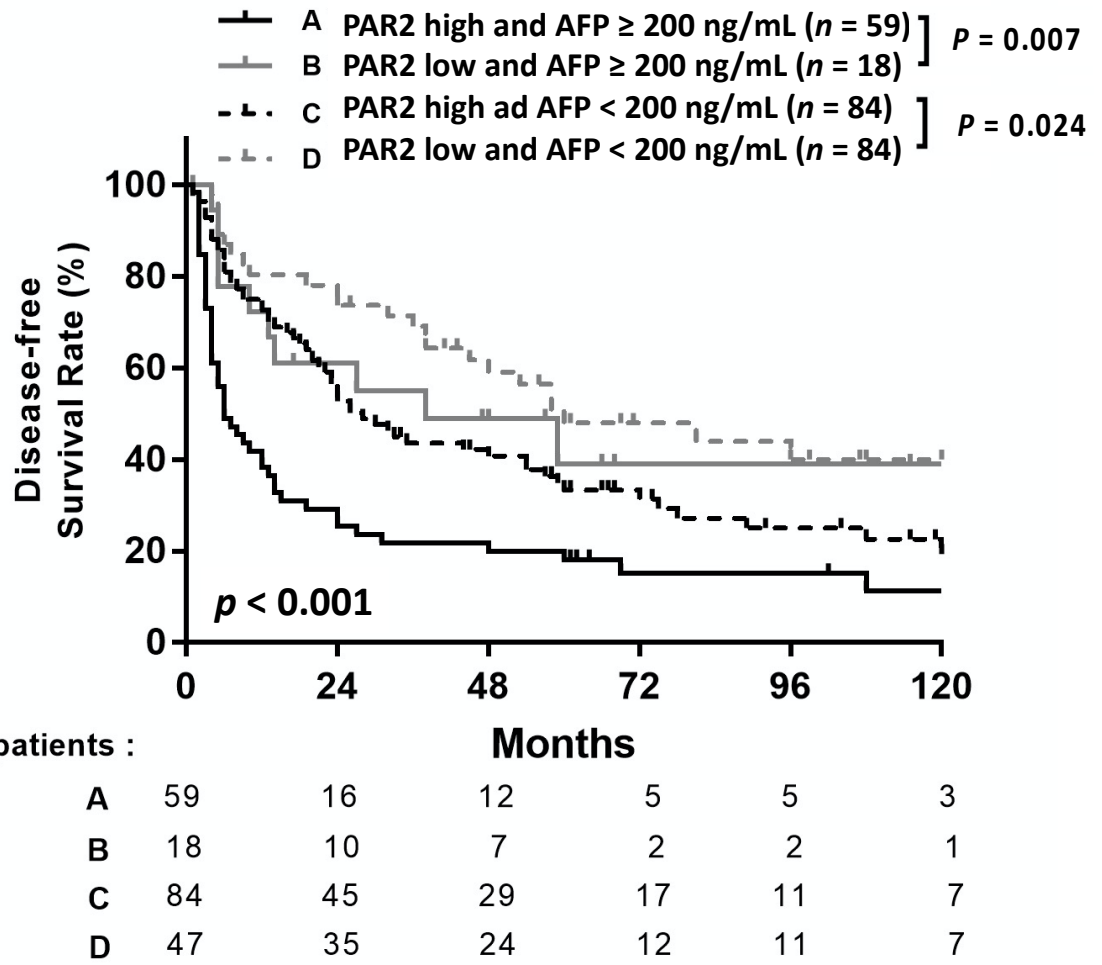

(A)

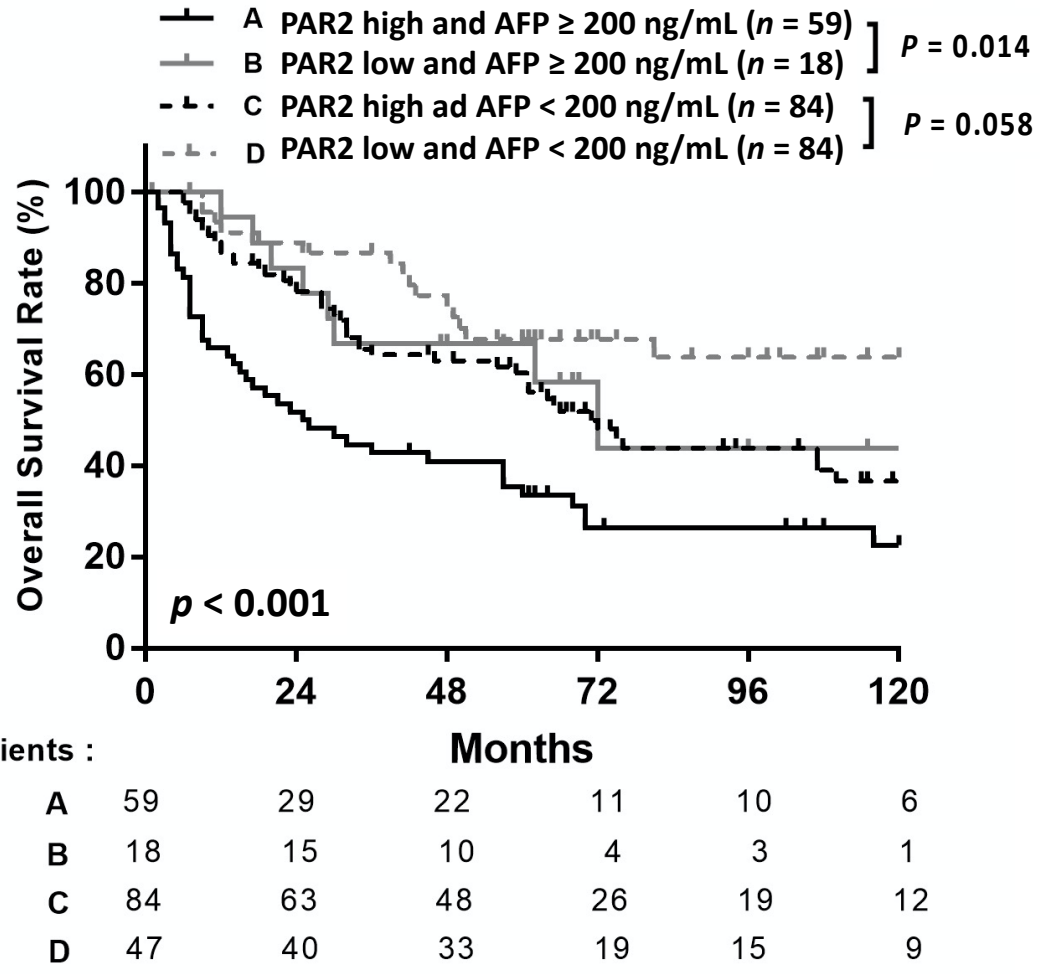

(B)

Figure 3. Disease-free survival (A) and overall survival (B) of HCC patients after resection stratified by the combination of PAR2 expression and AFP levels.

Our prior studies have demonstrated that coagulants tissue factor (TF) and factor VII (FVII) has a pathological role in promoting hepatoma growth by activating PAR2 [32,33]. We found that activation of TF/FVII/PAR2 axis is associated with increased invasiveness 
and migration in HCC cell lines in vitro, which is mainly via extracellular signal-regulated kinase-tuberous sclerosis complex (ERK-TSC). This mechanism could be explained by the increased expression of PAR2 in HCC tissues were associated with advanced TNM stage and poor DFS and OS. These findings were consistently observed in the study by Chen, in which PAR2 expression was increased in HBV-related HCC and high PAR2 expression was correlated with both poor DFS and OS [34].

In addition to PAR2, we found that serum AFP, cirrhosis, and advanced TNM stage were independent risk factors for recurrence and advanced TNM and histological grade were independent risk factors for OS. These data are in agreement with reports that tumorrelated factors may determine the outcomes of patients with HCC after resection [35,36]. AFP is a valuable, cost-effective serum biomarker used to assess the prognosis of AFPpositive patients with HCC in clinical practice. To explore the impact of serum AFP on DFS and OS in more detail, we performed a subgroup analysis based on AFP and PAR2. Patients with high expression of PAR2 and high serum AFP levels had significantly poorer DFS and OS rates than the other subgroups. Thus, the combination of AFP with PAR2 may provide an accurate prognostic tool for HCC. It is worth noting that low expression of PAR2 identified patients with a good prognosis in terms of DFS and OS in both the high and low serum AFP subgroups. Future studies are required to validate the clinical value of PAR2 in patients with HCC undergoing other treatments, such as radiofrequency ablation, transcatheter arterial chemoembolization, target therapy, and immunotherapy.

Collectively, we propose that PAR2 levels in HCC tissues may provide prognostic value, which may be particularly useful in the prediction of HCC patients after resection. It might be practical to provide adjuvant therapy for HCC patients with higher PAR2 expression after resection, who had poorer DFS and OS rate. A recent study suggests that I-191 may be a valuable antagonist of human PAR2 in cancer cells [37]. Thus, further clinical trials with adjuvant therapy after HCC resection are needed to verify the prognostic efficacy of PAR2.

Our study had some potential limitations. First, this is a retrospective study with patients from a single tertiary medical center, some patients didn't return to our hospital for further follow-up or even died after the operation, which could lead to biases. Second, more than half of the patients in the study are HBV infection, which is different from Western countries. However, we believe this difference did not affect our result due to no significant differences of PAR2 expression between hepatitis B and C. Finally, further studies in health individuals, and patients with other liver diseases, such as alcoholic liver disease and metabolic associated fatty liver disease, should be done to validate the role of PAR2 in the general population.

\section{Conclusions}

In conclusion, this study confirms that PAR2 is upregulated in HCC and has prognostic value after resection of HCC. Combination of PAR2 and AFP could represent a potentially useful prognostic factor in HCC and pave the way for the identification of novel pharmacological agents that target PAR2.

Author Contributions: Concept and design: C.-C.H.; Manuscript writing: M.-C.T.; Correction and assembly of data: C.-C.L., D.-W.C., Y.-W.L., and Y.-J.W.; Data analysis and interpretation: Y.-H.Y., P.-Y.H., C.-C.Y., and C.-H.C. All authors have read and agreed to the published version of the manuscript.

Funding: This study received no specific grant from any funding agency in the public, commercial, or not-for-profit sectors.

Institutional Review Board Statement: This study was performed in accordance with the Declaration of Helsinki and approved by the Institutional Review Board of Chang Gung Memorial Hospital (IRB number: 201800049B0; Date: from August 2018 to July 2019).

Informed Consent Statement: The ethics committee waived the requirement for informed consent; all patient data were anonymized. 
Data Availability Statement: The data presented in this study are available on request form the corresponding authors. The data are not publicly available due to our Institutional Review Board regulation.

Acknowledgments: The study was supported by grants CMRPG8F06661-2 from Chang Gung Memorial Hospital, Taiwan. The authors would like to thank Chang Gung Medical Foundation Kaohsiung Chang Gung Memorial Hospital Biobank and Tissue Bank Core Lab (CLRPG8L0081) for the technical support. We also thank the Biostatistics Center, Kaohsiung Chang Gung Memorial Hospital, for the statistical work.

Conflicts of Interest: The authors of this article have no conflicts of interest to declare.

\section{References}

1. Villanueva, A. Hepatocellular Carcinoma. N. Engl. J. Med. 2019, 380, 1450-1462. [CrossRef]

2. Kulik, L.; El-Serag, H.B. Epidemiology and Management of Hepatocellular Carcinoma. Gastroenterology 2019, 156, 477-491 e1. [CrossRef] [PubMed]

3. Bruix, J.; Sherman, M. American Association for the Study of Liver D. Management of hepatocellular carcinoma: An update. Hepatology 2011, 53, 1020-1022. [CrossRef] [PubMed]

4. Hirokawa, F.; Hayashi, M.; Asakuma, M.; Shimizu, T.; Inoue, Y.; Uchiyama, K. Risk factors and patterns of early recurrence after curative hepatectomy for hepatocellular carcinoma. Surg. Oncol. 2016, 25, 24-29. [CrossRef] [PubMed]

5. Zheng, J.; Kuk, D.; Gonen, M.; Balachandran, V.P.; Kingham, T.P.; Allen, P.J.; D'Angelica, M.I.; Jarnagin, W.R.; DeMatteo, R.P. Actual 10-Year Survivors After Resection of Hepatocellular Carcinoma. Ann. Surg. Oncol. 2017, 24, 1358-1366. [CrossRef] [PubMed]

6. Tabrizian, P.; Jibara, G.; Shrager, B.; Schwartz, M.; Roayaie, S. Recurrence of hepatocellular cancer after resection: Patterns, treatments, and prognosis. Ann. Surg. 2015, 261, 947-955. [CrossRef]

7. Nystedt, S.; Emilsson, K.; Wahlestedt, C.; Sundelin, J. Molecular cloning of a potential proteinase activated receptor. Proc. Natl. Acad. Sci. USA 1994, 91, 9208-9212. [CrossRef]

8. Riewald, M.; Ruf, W. Orchestration of coagulation protease signaling by tissue factor. Trends Cardiovasc. Med. 2002, 12, 149-154. [CrossRef]

9. Bar-Shavit, R.; Maoz, M.; Kancharla, A.; Jaber, M.; Agranovich, D.; Grisaru-Granovsky, S.; Uzeily, B. Protease-activated receptors (PARs) in cancer: Novel biased signaling and targets for therapy. Methods Cell Biol. 2016, 132, 341-358.

10. Ossovskaya, V.S.; Bunnett, N.W. Protease-activated receptors: Contribution to physiology and disease. Physiol. Rev. 2004, 84, 579-621. [CrossRef]

11. Ramachandran, R.; Hollenberg, M.D. Proteinases and signalling: Pathophysiological and therapeutic implications via PARs and more. Br. J. Pharmacol. 2008, 153 (Suppl 1), S263-S282. [CrossRef]

12. Kaufmann, R.; Oettel, C.; Horn, A.; Halbhuber, K.J.; Eitner, A.; Krieg, R.; Katenkamp, K.; Henklein, P.; Westermann, M.; Böhmer, F.D.; et al. Met receptor tyrosine kinase transactivation is involved in proteinase-activated receptor-2-mediated hepatocellular carcinoma cell invasion. Carcinogenesis 2009, 30, 1487-1496. [CrossRef] [PubMed]

13. Kaufmann, R.; Mussbach, F.; Henklein, P.; Settmacher, U. Proteinase-activated receptor 2-mediated calcium signaling in hepatocellular carcinoma cells. J. Cancer Res. Clin. Oncol. 2011, 137, 965-973. [CrossRef] [PubMed]

14. European Association for The Study of The Liver; European Organisation for Research and Treatment of Cancer. Treatment of C. EASL-EORTC clinical practice guidelines: Management of hepatocellular carcinoma. J. Hepatol. 2012, 56, 908-943. [CrossRef] [PubMed]

15. Bruix, J.; Sherman, M. Practice Guidelines Committee AAftSoLD. Management of hepatocellular carcinoma. Hepatology 2005, 42, 1208-1236. [CrossRef] [PubMed]

16. Kudo, M.; Izumi, N.; Kokudo, N.; Matsui, O.; Sakamoto, M.; Nakashima, O.; Kojiro, M.; Makuuchi, M.; HCC Expert Panel of Japan Society of Hepatology. Management of hepatocellular carcinoma in Japan: Consensus-Based Clinical Practice Guidelines proposed by the Japan Society of Hepatology (JSH) 2010 updated version. Dig. Dis. 2011, 29, 339-364. [CrossRef] [PubMed]

17. Omata, M.; Lesmana, L.A.; Tateishi, R.; Chen, P.J.; Lin, S.M.; Yoshida, H.; Kudo, M.; Lee, J.M.; Choi, B.I.; Poon, R.T.; et al. Asian Pacific Association for the Study of the Liver consensus recommendations on hepatocellular carcinoma. Hepatol. Int. 2010, 4, 439-474. [CrossRef]

18. Surveillance Group; Diagnosis Group; Staging Group; Surgery Group; Local Ablation Group; TACE/TARE/HAI Group; Target Therapy/Systemic Therapy Group; Radiotherapy Group; Prevention Group; Drafting Group. Management consensus guideline for hepatocellular carcinoma: 2016 updated by the Taiwan Liver Cancer Association and the Gastroenterological Society of Taiwan. J. Formos. Med. Assoc. 2018, 117, 381-403. [CrossRef]

19. Edge, S.B.; Compton, C.C. The American Joint Committee on Cancer: The 7th edition of the AJCC cancer staging manual and the future of TNM. Ann. Surg. Oncol. 2010, 17, 1471-1474. [CrossRef]

20. Edmondson, H.A.; Steiner, P.E. Primary carcinoma of the liver: A study of 100 cases among 48,900 necropsies. Cancer 1954, 7, 462-503. [CrossRef] 
21. Everhart, J.E.; Wright, E.C.; Goodman, Z.D.; Dienstag, J.L.; Hoefs, J.C.; Kleiner, D.E.; Ghany, M.G.; Mills, A.S.; Nash, S.R.; Govindarajan, S.; et al. Prognostic value of Ishak fibrosis stage: Findings from the hepatitis C antiviral long-term treatment against cirrhosis trial. Hepatology 2010, 51, 585-594. [CrossRef] [PubMed]

22. Espinosa, W.; Liu, Y.W.; Wang, C.C.; Lin, C.C.; Wang, J.H.; Lu, S.N.; Huang, H.C. Combined resection and radiofrequency ablation versus transarterial embolization for intermediate-stage hepatocellular carcinoma: A propensity score matching study. J. Formos. Med. Assoc. 2018, 117, 197-203. [CrossRef]

23. Adams, M.N.; Ramachandran, R.; Yau, M.K.; Suen, J.Y.; Fairlie, D.P.; Hollenberg, M.D.; Hooper, J.D. Structure, function and pathophysiology of protease activated receptors. Pharmacol. Ther. 2011, 130, 248-282. [CrossRef]

24. Ramachandran, R.; Noorbakhsh, F.; Defea, K.; Hollenberg, M.D. Targeting proteinase-activated receptors: Therapeutic potential and challenges. Nat. Rev. Drug Discov. 2012, 11, 69-86. [CrossRef] [PubMed]

25. Su, X.; Matthay, M.A. Role of protease activated receptor 2 in experimental acute lung injury and lung fibrosis. Anat. Rec. 2009, 292, 580-586. [CrossRef] [PubMed]

26. Fujimoto, D.; Hirono, Y.; Goi, T.; Katayama, K.; Hirose, K.; Yamaguchi, A. Expression of protease activated receptor-2 (PAR-2) in gastric cancer. J. Surg. Oncol. 2006, 93, 139-144. [CrossRef]

27. Jahan, I.; Fujimoto, J.; Alam, S.M.; Sato, E.; Sakaguchi, H.; Tamaya, T. Role of protease activated receptor-2 in tumor advancement of ovarian cancers. Ann. Oncol. 2007, 18, 1506-1512. [CrossRef]

28. Zhang, X.; Wang, W.; Mize, G.J.; Takayama, T.K.; True, L.D.; Vessella, R.L. Protease-activated receptor 2 signaling upregulates angiogenic growth factors in renal cell carcinoma. Exp. Mol. Pathol. 2013, 94, 91-97. [CrossRef]

29. Chang, J.H.; Park, J.M.; Kim, S.W.; Jung, C.K.; Kang, W.K.; Oh, S.T. Expression of protease activated receptor-2 in human colorectal cancer and its association with tumor progression. Dis. Colon Rectum 2010, 53, 1202-1208. [CrossRef]

30. Ungefroren, H.; Witte, D.; Rauch, B.H.; Settmacher, U.; Lehnert, H.; Gieseler, F.; Kaufmann, R. Proteinase-Activated Receptor 2 May Drive Cancer Progression by Facilitating TGF-beta Signaling. Int. J. Mol. Sci. 2017, 18, 2494. [CrossRef]

31. Wojtukiewicz, M.Z.; Hempel, D.; Sierko, E.; Tucker, S.C.; Honn, K.V. Protease-activated receptors (PARs)-biology and role in cancer invasion and metastasis. Cancer Metastasis Rev. 2015, 34, 775-796. [CrossRef]

32. Chen, K.D.; Huang, K.T.; Tsai, M.C.; Wu, C.H.; Kuo, I.Y.; Chen, L.Y.; Lin, C.C. Coagulation factor VII and malignant progression of hepatocellular carcinoma. Cell Death Dis. 2016, 7, e2110. [CrossRef] [PubMed]

33. Tsai, M.C.; Chen, K.D.; Wang, C.C.; Huang, K.T.; Wu, C.H.; Kuo, I.Y.; Chen, L.Y.; Hu, T.H.; Goto, S.; Nakano, T.; et al. Factor VII promotes hepatocellular carcinoma progression through ERK-TSC signaling. Cell Death Discov. 2015, 1, 15051. [CrossRef] [PubMed]

34. Chen, P.; Yang, N.; Xu, L.; Zhao, F.; Zhang, M. Increased expression of protease-activated receptors 2 indicates poor prognosis in HBV related hepatocellular carcinoma. Infect. Agent Cancer 2019, 14, 39. [CrossRef] [PubMed]

35. Chan, A.W.H.; Zhong, J.; Berhane, S.; Toyoda, H.; Cucchetti, A.; Shi, K.; Tada, T.; Chong, C.C.N.; Xiang, B.D.; Li, L.Q.; et al. Development of pre and post-operative models to predict early recurrence of hepatocellular carcinoma after surgical resection. $J$. Hepatol. 2018, 69, 1284-1293. [CrossRef] [PubMed]

36. Chou, W.C.; Lee, C.L.; Yang, T.S.; Huang, C.Y.; Teng, W.; Tseng, Y.T.; Chen, J.S.; Lin, Y.C.; Hou, M.M.; Chang, H.H.; et al. Changes in serum alpha-fetoprotein level predicts treatment response and survival in hepatocellular carcinoma patients and literature review. J. Formos. Med. Assoc. 2018, 117, 153-163. [CrossRef] [PubMed]

37. Jiang, P.; De Li, S.; Li, Z.G.; Zhu, Y.C.; Yi, X.J.; Li, S.M. The expression of protease-activated receptors in esophageal carcinoma cells: The relationship between changes in gene expression and cell proliferation, apoptosis in vitro and growing ability in vivo. Cancer Cell Int. 2018, 18, 81. [CrossRef] [PubMed] 